$\begin{array}{rr}\text { Universitas } & \text { Vol. } 37 \\ \text { Núm. } 78\end{array}$

\title{
Frecuencia, hallazgos y variaciones óseas en radiografías panorámicas de personas con edentulismo total ${ }^{*}$
}

Frequency, Findings, and Bone Variations in Panoramic Radiographs among People with Total Edentulism

\author{
Johan Escolano Rivas \\ Pontificia Universidad Javeriana, Colombia \\ ORCID: http://orcid.org/0000-0001-8077-2796
}

Silvia Barrientos Sánchez

Pontificia Universidad Javeriana, Colombia

ORCID: http://orcid.org/0000-0001-7280-3791

Adriana Rodríguez Ciódaro ${ }^{\text {a }}$
Pontificia Universidad Javeriana, Colombia
arodrig@javeriana.edu.co
ORCID: http://orcid.org/0000-0002-6640-3975
DOI: https://doi.org/10.11144/Javeriana.uo37-78.fhvo Redalyc: http://www.redalyc.org/articulo.oa? id $=231260072002$

Fecha de recepción: 04 Junio 2018 Fecha de aprobación: 11 Junio 2018 Fecha de publicación: 18 Junio 2018

\section{Resumen:}

Antecedentes: Según el Estudio Nacional de Salud Bucal de 2014, el 5,20 \% de la población colombiana presenta edentulismo total, $32,8 \%$ de los cuales son personas mayores de 65 años de edad; aunque se pueden realizar análisis radiográficos para reconocer otras características relacionadas con el edentulismo. Objetivo: Identificar la frecuencia, las características y las variaciones óseas de pacientes totalmente edéntulos en radiografías panorámicas. Métodos: Se realizó un estudio observacional descriptivo en una población de 10.000 radiografías panorámicas entre las cuales se identificaron, mediante el programa CliniView 9.3, las que correspondían a pacientes con edentulismo total. Se registraron variables como sexo, edad, altura de rebordes alveolares, altura de rama y cóndilo mandibulares y otros hallazgos radiográficos. Resultados: Se encontraron 112 radiografías de pacientes con edentulismo total, $51,8 \%$ de las cuales tenía algún tipo de hallazgo radiográfico. Los más frecuentes fueron implantes (33,9\%) y restos radiculares $(13,4 \%)$. No hubo diferencias en los promedios de las medidas óseas en los lados derecho e izquierdo. La altura del reborde alveolar en el maxilar inferior fue mayor en hombres que en mujeres $(p<0,05)$. Los valores de altura condilar fueron similares en ambos sexos. Se encontró mayor la altura de la rama mandibular en hombres $(\mathrm{p}<0,05)$. Conclusiones: La frecuencia de hallazgos radiográficos en pacientes edéntulos totales es considerable. Las variaciones óseas relacionadas con la ausencia de dientes deben tenerse en cuenta para tomar decisiones clínicas de restauración con prótesis total o con implantes.

Palabras clave: diagnóstico, edentulismo total, odontología, patología oral, radiografía panorámica, radiología.

\begin{abstract}
:
Background: According to the 2014 National Oral Health Study, 5,20\% of the Colombian population suffer from total edentulism, $32,8 \%$ of them were 65 years or older. Other characteristics associated with edentulism could be recognized through radiographic analysis. Purpose: To identify the frequency, characteristics, and bone variations of totally edentulous patients in panoramic radiographs. Methods: The population of this descriptive, observational study consisted of 10.000 panoramic radiographs, from which those corresponding to patients with total edentulism were analyzed using the CliniView 9.3 program. Variables such as age, alveolar ridge height, ramus height and condyle, and radiographic findings were recorded. Results: 112 radiographs of patients with total edentulism were found, of which $51,8 \%$ showed radiographic findings. The most frequent findings were implants (33,9\%) and root remains in $(13,4 \%)$. There were no differences regarding bone measurement averages between the right and left sides. Men showed higher alveolar ridge height in the mandible than women $(p<0,05)$. Condylar height values were similar in both sexes. Mandibular ramus height was larger among men than women $(p<0,05)$. Conclusions: Frequency of radiographic findings in total edentulous patients is considerable. Bone variations related to tooth absence must be taken into account when making restorative decisions with total prosthesis and/or implants.
\end{abstract}

Keywords: dentistry, diagnosis, oral pathology, panoramic X-ray, radiology, total edentulism.

Notas de autor:

a Autora de correspondencia. Correo electrónico: arodrig@javeriana.edu.co 


\section{Introducción}

El IV Estudio Nacional de Salud Bucal (ENSAB IV) (1) encontró que el 5,20 \% de la población en Colombia ha perdido la totalidad de sus dientes. El porcentaje de edentulismo es del 0,5\% a los 35 años de edad, se incrementa al 7,43\% entre los 45 y los 64 años y alcanza el 32,87 \% entre los 65 y los 79 años. A pesar de la alta prevalencia de la pérdida dental, aun a temprana edad, no hay estudios poblaciones que muestren las patologías dentales yóseas que se encuentran en esta población y que solo se pueden analizar sobre radiografías como las panorámicas.

Teniendo en cuenta que la pérdida de los dientes modifica la anatomía ósea y que las patologías asociadas son generalmente asintomáticas, en términos generales se recomienda usar radiografías panorámicas, incluso en pacientes edéntulos asintomáticos. Las razones para recomendar análisis radiográficos incluyen alto valor diagnóstico, relación costo-beneficio, poca radiación y visualización completa de los maxilares $(2,3)$ y estructuras anatómicas relacionadas (4). Rivas y colaboradores (5) sugieren que sería adecuado solicitar una radiografía panorámica antes de iniciar el tratamiento rehabilitador de un paciente adulto mayor para obtener información que permita un tratamiento odontológico de forma segura.

Con respecto a las patologías asociadas, Bohay y colaboradores (2), en un estudio con una muestra de 375 personas con edentulismo total, encontraron que en el 7,3\% de los casos se presentaron restos radiculares, dientes incluidos y crestas alveolares atróficas. Kose y colaboradores (3) hallaron que en el $34 \%$ de los hallazgos radiográficos en edéntulos asintomáticos se encontraban restos radiculares (9,4\%), dientes impactados $(4,8 \%)$, cuerpos extraños $(2,2 \%)$ y quistes $(6,3 \%)$. Tales condiciones generan decisiones particulares de tratamiento, pues en un $54 \%$ de los casos se requieren procedimientos quirúrgicos previos a la colocación de implantes o a la confección de prótesis totales. Adicionalmente, se ha observado que la pérdida de dientes en el maxilar superior se asocia con neumatización sinusal y se necesita cirugía de elevación de seno en más de la mitad de los casos (6).

El objetivo de este estudio fue identificar la frecuencia, las características y las variaciones óseas de pacientes totalmente edéntulos en radiografías panorámicas, con el fin de complementar la información registrada en el ENSAB IV (1) sobre tendencias de las patologías y las alteraciones relacionadas con el edentulismo total.

\section{Materiales y métodos}

Se realizó un estudio descriptivo de corte transversal a partir de una población de 10.000 radiografías de diferentes centros radiológicos de la ciudad de Bogotá, Colombia. El estudio recibió aprobación del Comité de Investigación y Ética de la Facultad de Odontología de la Pontificia Universidad Javeriana.

Se incluyeron radiografías panorámicas digitales completas, con adecuada densidad y contraste, correspondientes a pacientes edéntulos totales bimaxilares, con imagen completa de los maxilares y estructuras vecinas. Se registraron los datos de sexo, edad y hallazgo radiográfico en una hoja de Microsoft Excel $^{\circ}$. Un residente de cirugía maxilofacial evaluó todas las radiografías bajo la supervisión de una estomatóloga experta en radiología. Para identificar las alteraciones, anomalías y procesos patológicos, denominados hallazgos radiográficos bucales, se analizaron de manera sistemática los 4 cuadrantes. Se registró la presencia de hallazgos patológicos (lesiones radiolúcidas/radiopacas compatibles con quistes y tumores, fracturas, restos radiculares y dientes incluidos) y no patológicos (implantes, material de osteosíntesis y cuerpos extraños). La medición de las alturas de los rebordes alveolar superior e inferior, así como de la rama y el cóndilo mandibular, se realizó con el programa CliniView versión 9.3 (Instrumentarium Dental, Milwaukee, Estados Unidos), localizado en la sala de radiología de la Facultad de Odontología, por medio de los puntos de referencia que se muestran en la figura 1. Para medir la altura del reborde alveolar se tomaron medidas en 3 puntos en el maxilar superior (derecho, espina nasal e izquierdo) y 3 en el maxilar 
inferior (derecho, sínfisis e izquierdo), así como las alturas de rama y de cóndilo. Para valorar los hallazgos del seno maxilar se utilizó la clasificación de Misch (7). Se tomó como criterio para definir un seno maxilar neumatizado aquel que presentara una clasificación de Misch grado II, es decir, que su reborde alveolar no fuera mayor a $8 \mathrm{~mm}$, que es el límite para la colocación de implantes sin elevación del piso del seno maxilar.

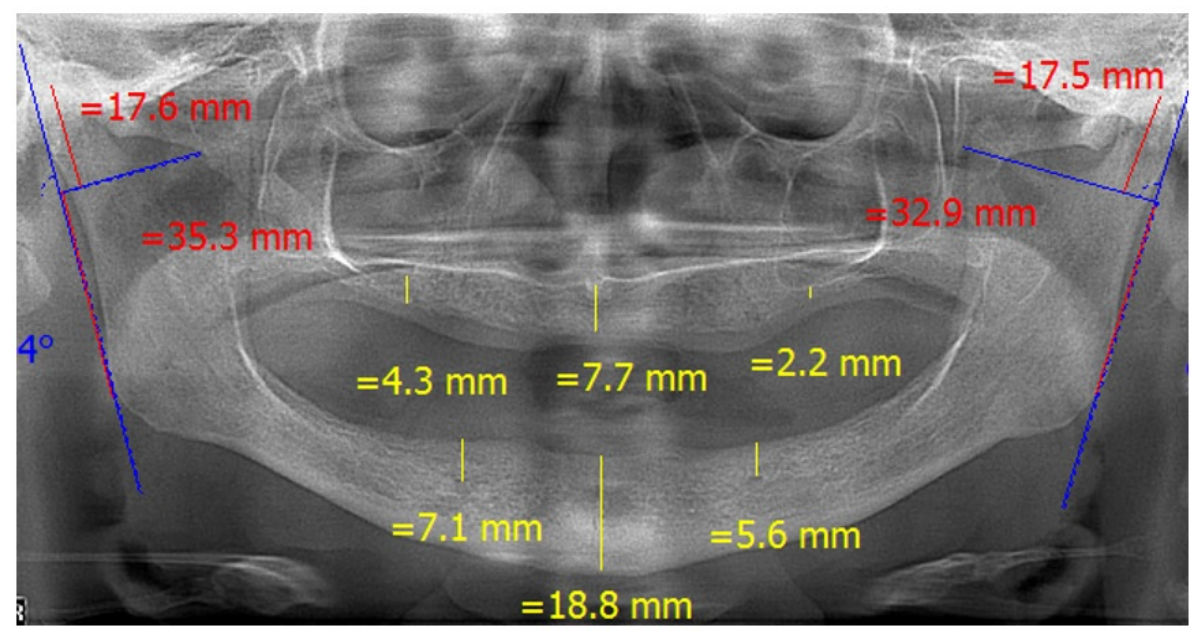

FIGURA 1

Puntos de medición de las alturas de los rebordes superior e inferior y alturas de rama y cóndilo en radiografías de edéntulos totales

Fuente: elaboración propia.

Para analizar descriptivamente los hallazgos se usaron frecuencias absolutas y relativas, medidas de tendencia central y de dispersión de cada una de las variables estudiadas. Las variables cuantitativas se compararon por medio de la prueba $\mathrm{T}$, con una significancia del $95 \%$.

\section{Resultados}

Del grupo de 10.000 radiografías panorámicas revisadas se obtuvieron 112 radiografías de pacientes edéntulos totales (1,12\%), de los cuales el 70,5\% eran mujeres y el 29,5\% de hombres. El promedio de edad era de 65,8 años con un rango de 41 a 91 años. La distribución por edad mostró una frecuencia del $8 \%$ ( 9 casos) de personas de 50 años o menos, del $26 \%$ (29 casos) de 51 a 60 años, del 27,7\% (31 casos) de 61 a 70 años, del 29,5\% (33 casos) de 71 a 80 años y del $9 \%$ (10 casos) mayores de 81 años.

Se encontró algún tipo de hallazgo radiográfico en el 51,8 \% (58 casos) de las 112 radiografías. Los más frecuentes fueron implantes $(33,9 \%)$ y restos radiculares $(13,4 \%)$. Los otros hallazgos fueron presencia de cuerpo extraño $(5,4 \%)$, diente incluido (4,5\%), material de osteosíntesis (1,79\%), zona radiolúcida compatible con lesión quística $(0,9 \%)$ y zona radiopaca compatible con tumor benigno (0,9\%) (figura 2 ). 


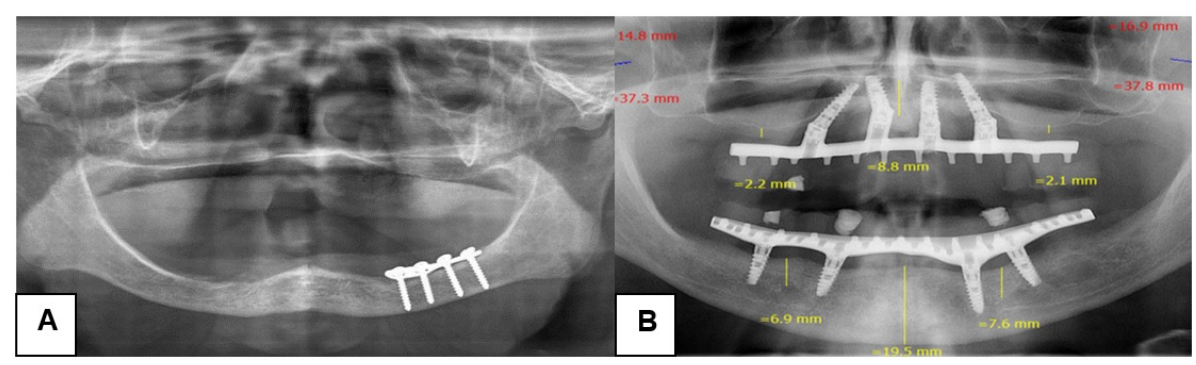

FIGURA 2

Hallazgos radiográficos en los maxilares de pacientes con edentulismo total

a) Fractura mandibular. b) Implantes dentales Fuente: elaboración propia.

En las 38 imágenes con implantes se observaron 239 implantes convencionales, en un rango de 1 a 19 implantes por paciente. En 3 radiografías se observaron 4 implantes cigomáticos; en 8 se presentó la configuración de All-on- $4^{\oplus}$ de los implantes y al menos 3 implantes se encontraban en mala posición.

En relación con los hallazgos en el seno maxilar, 92 pacientes ( $82 \%)$ presentaron neumatización de al menos uno de sus maxilares sin preferencia por la edad, el lado o el sexo. El 1,8 \% (2 casos) presentó una comunicación bucosinusal, un resto radicular $(0,9 \%)$, un molar $(0,9 \%)$ y una maxilectomía $(0,9 \%)$ (figura 3 ).

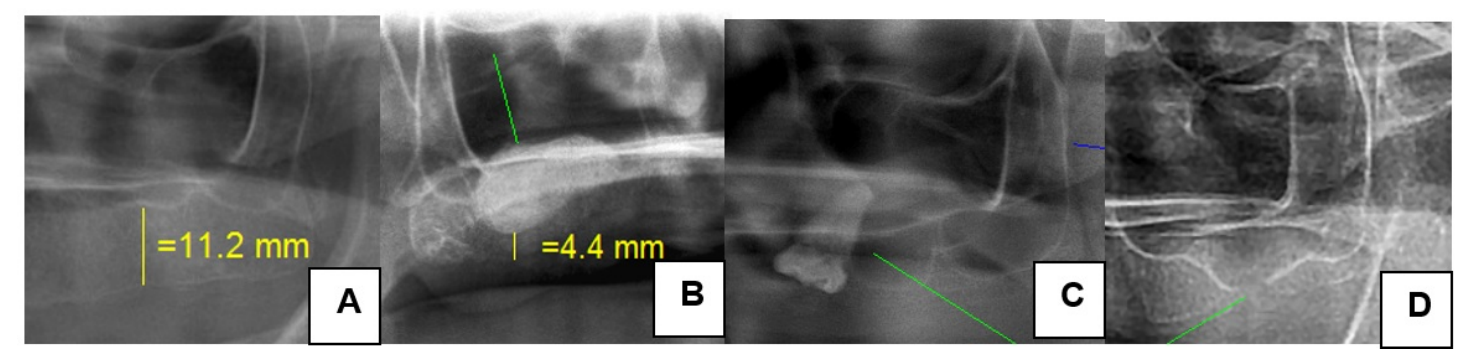

FIGURA 3

Imágenes de hallazgos radiográficos en los senos maxilares

a) Normal. b) Neumatizado y con presencia de injerto óseo. c) Presencia de molar en el seno maxilar. d) Comunicación bucosinusal Fuente: elaboración propia.

El promedio de las medidas de la altura del reborde alveolar superior fue de $6,5 \mathrm{~mm}$; mientras que en el reborde inferior este fue de $11,1 \mathrm{~mm}$. Los valores para cada uno de los 3 puntos medidos se muestran en la tabla 1. El análisis comparativo no mostró diferencias entre los dos lados (derecho e izquierdo) de la altura de reborde superior e inferior, la altura de los cóndilos y de las ramas mandibulares. 
TABLA 1

Valores de las alturas de rebordes alveolares, cóndilos y rama mandibular en pacientes con edentulismo total

\begin{tabular}{lrrr}
\hline \multirow{2}{*}{\multicolumn{1}{c}{ Lugar de medición }} & \multicolumn{2}{c}{$\begin{array}{c}\text { Valores promedio }(\mathrm{mm}) \\
\pm \text { desviación estándar }\end{array}$} \\
\cline { 2 - 4 } & Hombres & \multicolumn{1}{c}{ Mujeres } & Total \\
\hline Reborde superior derecho & $4,7 \pm 3,1$ & $5,2 \pm 3,1$ & $5,1 \pm 3,1$ \\
\hline Reborde superior espina nasal & $9,6 \pm 3,3$ & $8,8 \pm 2,9$ & $9,0 \pm 3,0$ \\
\hline Reborde superior izquierdo & $5,1 \pm 3,2$ & $5,2 \pm 2,9$ & $5,1 \pm 2,9$ \\
\hline Reborde inferior derecho & $7,4 \pm 4,2$ & $5,4 \pm 4,1$ & $6,0 \pm 4,2$ \\
\hline Reborde inferior sínfisis & $22,2 \pm 4,7$ & $20,1 \pm 5,3$ & $20,8 \pm 5,2$ \\
\hline Reborde inferior izquierdo & $7,2 \pm 4,7$ & $5,4 \pm 4,0$ & $6,0 \pm 4,3$ \\
\hline Cóndilo derecho & $19,0 \pm 3,9$ & $19,1 \pm 3,9$ & $19,0 \pm 3,9$ \\
\hline Cóndilo izquierdo & $18,4 \pm 3,1$ & $18,9 \pm 4,2$ & $18,7 \pm 3,9$ \\
\hline Rama mandibular derecha & $37,6 \pm 6,2$ & $33,8 \pm 5,5$ & $34,9 \pm 6,0$ \\
\hline Rama mandibular izquierda & $37,5 \pm 6,5$ & $33,8 \pm 5,6$ & $35,0 \pm 6,1$ \\
\hline
\end{tabular}

Fuente: elaboración propia.

La comparación por sexo mostró una mayor altura de reborde alveolar en el maxilar inferior en los hombres que las mujeres, en los tres sitios de donde se obtuvo la información (derecho: $\mathrm{p}=0,02$; sínfisis: $\mathrm{p}=0,03$; e izquierdo: $p=0,03$ ). Esta situación no se observó en el maxilar superior. Los valores de altura condilar fueron similares para ambos sexos en ambos lados. Se encontró un valor significativamente mayor de la altura de la rama en los hombres (derecha: $\mathrm{p}=0,004$; izquierda: $\mathrm{p}=0,007$ ) que en las mujeres (tabla 1 ).

\section{Discusión}

El valor diagnóstico de la radiografía panorámica en pacientes edéntulos totales es considerable. Permite evaluar o determinar reabsorción ósea, neumatización de los senos maxilares, síndrome de Eagle en pacientes de la tercera edad, ateromas calcificados de la arteria carótida, densidad ósea, calcificaciones, dientes impactados, restos radiculares, cuerpos extraños, lesiones tumorales y quísticas y fracturas óseas $(8,9,10)$. Los resultados del presente estudio mostraron un $1,12 \%$ de personas con edentulismo total, identificado a través de análisis radiográfico. Este valor es menor al 5,2 \% reportado en el ENSAB IV, aunque era esperable teniendo en cuenta que la metodología usada en dicha encuesta (1) fue examen clínico en población general con acceso a los servicios de salud y sin este.

Como en la mayoría de los estudios epidemiológicos, los resultados pueden variar de acuerdo con la población estudiada. La frecuencia total de los hallazgos radiográficos en este estudio fue similar a lo descrito por Rivas y colaboradores (5) en población chilena y Masood y colaboradores (9) en Oklahoma, pero hay variaciones en las frecuencias según el tipo de hallazgo reportado. Es interesante anotar que Rivas y colaboradores (5) muestran un $20 \%$ de lesiones radiopacas, un valor significativamente mayor que en este estudio, en el que se encontró solo un caso $(0,9 \%)$.

Las alteraciones de los senos maxilares en pacientes edéntulos pueden requerir procedimientos quirúrgicos, casos en los que la imagen es necesaria para buscar patologías o para evaluar la necesidad de una 
elevación del piso del seno maxilar para la colocación de implantes (11). Las alturas alveolares son un parámetro que se debe considerar en el momento de rehabilitar pacientes edéntulos, ya que los rebordes atróficos afectan negativamente la estabilidad de las prótesis mucosoportadas, principalmente la mandibular. Igualmente, los rebordes atróficos en altura impiden la colocación de implantes convencionales para prótesis implantosoportadas, lo que hace necesario utilizar técnicas alternativas como All-on-4, regeneraciones óseas, elevación del seno maxilar e implantes cigomáticos (12).

La reabsorción del reborde óseo maxilar y mandibular es una consecuencia del edentulismo total. El hueso disminuye en cantidad y calidad a partir de la tercera década de la vida. Además, la pérdida prematura de dientes induce la reabsorción de la cresta alveolar circundante ósea, seguida de remodelación y que finalmente conduce a la atrofia de los rebordes desdentados. Si bien el grado de reabsorción ósea del reborde alveolar depende principalmente del tiempo transcurrido desde la pérdida dental, existen otras variables que inciden, como la edad y el sexo (13). En el presente estudio se mostró una mayor altura del reborde alveolar mandibular en hombres con edentulismo que en mujeres, situación que no se observó en el maxilar superior. El estudio de Panchbhai (14) también encontró una reducción en la altura del reborde entre $29 \%$ y el $39 \%$, con mayor porcentaje en la mandíbula que en el maxilar, y una mayor reabsorción en las mujeres que en los hombres. De igual manera, Yüzügüllü y colaboradores (15) en población turca y Xie y colaboradores (16) hallaron una mayor reabsorción del reborde alveolar mandibular en las mujeres.

Otro factor que puede generar cambios en las características faciales de los pacientes edéntulos es la variación del tamaño de los cóndilos y la rama mandibular. El estudio de Joo y colaboradores (17) analizó los cambios en la altura de la rama y la altura del cóndilo en relación con el sexo. Concluyó que las mujeres sufren cambios morfológicos más evidentes debido al edentulismo que los hombres, como se muestra en el presente estudio en el que la altura de la rama mandibular es significativamente mayor en hombres que en mujeres. La comparación con los valores encontrados en pacientes dentados en esta misma población y usando la misma metodología de medición (18) indica una pérdida en la altura de la rama mandibular de un $40 \%$ aproximadamente en mujeres y de $36 \%$ en hombres con edentulismo total. Ello conduce a una disminución vertical del perfil del paciente que genera el rasgo clásico de envejecimiento y altera el funcionamiento de la articulación temporomandibular. Respecto de la altura de los cóndilos, no se encontraron diferencias significativas entre ambos sexos y el promedio fue aproximadamente un $16 \%$ mayor en los pacientes edéntulos comparados con los pacientes con dentición completa (19).

El edentulismo total conduce a una serie de afecciones que repercuten en la calidad de vida de los pacientes. Los rebordes atróficos impiden una correcta rehabilitación ya sea por medio de prótesis mucosoportadas o implantosoportadas, cambios importantes en el perfil facial que generan la apariencia de vejez en las personas afectadas y, sobre todo, una disminución en la ingesta de una dieta calórica, debido a las limitaciones para ingerir diferentes tipos de alimentos. Además, en Colombia el 10,5 \% de la población es mayor de 60 años y se espera un aumento de esta proporción en los próximos años (19). Está claro que la mejor política de salud es la conservación de los dientes en boca el mayor tiempo posible, por lo que se debe enfatizar la necesidad de programas de prevención adecuados y apropiados para la población de la tercera edad en creciente aumento, así como del manejo de estos pacientes mediante una rehabilitación acorde a sus necesidades.

\section{Conclusiones}

Se encontraron hallazgos radiográficos en el 51,8 \% de las radiografías panorámicas de personas con edentulismo total.

El hallazgo más frecuente fueron los implantes dentales.

La frecuencia de lesiones óseas compatibles con quistes y tumores fue baja.

El $82 \%$ de los pacientes edéntulos presentaba neumatización del seno maxilar. 
Los cambios ocasionados por la pérdida de los dientes afectan más las condiciones óseas de las mujeres.

\section{Recomendaciones}

Diseñar nuevos estudios que contribuyan al conocimiento del paciente edéntulo, utilizando tomografías. Evaluar las modificaciones de otras estructuras anatómicas como la articulación temporomandibular y los tejidos blandos ocasionadas por la pérdida de los dientes.

\section{Referencias}

1. Ministerio de Salud. Cuarto Estudio Nacional de Salud Bucal (ENSAB IV). Bogotá, Colombia: Ministerio de Salud; 2014.

2. Bohay RN, Stephens RG, Kogon SL. A study of the impact of screening or selective radiography on the treatment and postdelivery outcome for edentulous patients. Oral Surg Oral Med Oral Pathol Oral Radiol Endod. 1998 Sep; 86(3): 353-9. https://doi.org/10.1016/S1079-2104(98)90185-8

3. Kose TE, Demirtas N, Cakir KH, Ozcan I. Evaluation of dental panoramic radiographic findings in edentulous jaws: A retrospective study of 743 patients "Radiographic features in edentulous jaws". J Adv Prosthodont. 2015 Oct; 7(5): 380-5. https://doi.org/10.4047/jap.2015.7.5.380

4. Choi J. Assessment of panoramic radiography as a national oral examination tool: review of the literature. Imaging Sci Dent. 2011 Mar; 41(11): 1-6. https://doi.org/10.5624/isd.2011.41.1.1

5. Rivas H, Eymin Q, Quezada S. Hallazgos en radiografías panorámicas de adultos mayores de Talca, Chile. Acta Odontol Venez [Internet]. 2013 Abr-Jun; 51(2). Disponible en: https://www.actaodontologica.com/edicione s/2013/2/art-6/

6. Cavalcanti MC, Guirado TE, Sapata VM, Costa C, Pannuti CM, Jung RE, César Neto J. Maxillary sinus floor pneumatization and alveolar ridge resorption after tooth loss: a cross-sectional study. Braz Oral Res. 2018 Aug 6;32:e64. https://doi.org/10.1590/1807-3107BOR-2018.vol32.0064

7. Misch CE. Maxillary sinus augmentation for endosteal implants: organized alternative treatment plans. Int J Oral Implantol. 1987; 4(2): 49-58.

8. Awad E, Al-Dharrab A. Panoramic radiographic examination: a survey of 271 edentulous patients. Int $J$ Prosthodont. 2011 Jan-Feb; 24(1): 55-7.

9. Masood F, Robinson W, Beavers KS, Haney KL. Findings from panoramic radiographs of the edentulous population and review of the literature. Quintessence Int. 2007 Jun; 38(6): e298-305.

10. Bodner L, Manor E, Glazer M, Brennan PA. Cystic lesions of the jaws in edentulous patients: analysis of 27 cases. Br J Oral Maxillofac Surg. 2011 Dec; 49(8): 643-46. https://doi.org/10.1016/j.bjoms.2010.10.009

11. Manji A, Faucher J, Resnik R, Suzuki JB. Prevalence of maxillary sinus pathology in patients considered for sinus augmentation procedures for dental implants. Implant Dent. 2013 Aug; 22(4): 428-35. https://doi.org/10.10 97/ID.0b013e31829d1a20

12. Güler AU, Sumer M, Sumer P, Bicer I. The evaluation of vertical heights of maxillary and mandibular bones and the location of anatomic landmarks in panoramic radiographs of edentulous patients for implant dentistry. J Oral Rehabil. 2005 Oct; 32(10): 741-6.

13. Sairam V, Potturi GR, Praveen B, Vikas G. Assessment of effect of age, gender, and dentoalveolar changes on mandibular morphology: a digital panoramic study. Contemp Clin Dent. 2018 Jan-Mar; 9(1): 49-54.

14. Panchbhai A. Quantitative estimation of vertical heights of maxillary and mandibular jawbones in elderly dentate and edentulous subjects. Spec Care Dentist. 2013 Mar-Apr; 33(2): 62-9. https://doi.org/10.1111/j.1754-450 5.2012.00301.x 
15. Yüzügüllü B, Gulsahi A, Imirzalioglu P. Radiomorphometric indices and their relation to alveolar bone loss in completely edentulous Turkish patients: A retrospective study. J Prosthet Dent. 2009 Mar; 101(3): 160-5. http s://doi.org/10.1016/S0022-3913(09)60021-4

16. Xie Q, Wolf J, Tilvis R, Ainamo A. Resorption of mandibular canal wall in the edentulous aged population. J Prosthet Dent. 1997 Jun; 77(6): 596-600.

17. Joo JK Lim YJ, Kwon HB, Ahn SJ. Panoramic radiographic evaluation of the mandibular morphological changes in elderly dentate and edentulous subjects. Acta Odontol Scand. 2013 Mar; 71(2): 357-62. https://doi.org/10 $.3109 / 00016357.2012 .690446$

18. Alfaro C, Ayala R, Barrientos S. Rodríguez A. Prevalencia de asimetrías mandibulares en radiografías panorámicas en población de Bogotá Colombia. Int J Morphol. 2016 Oct; 34(4): 1203-6. http://dx.doi.org/10.4067/S071 7-95022016000400004

19. Ministerio de Salud y Protección Social. Envejecimiento demográfico. Colombia 1951-2020: Dinámica demográfica y estructuras poblacionales. Bogotá, Colombia: Ministerio de Salud y Protección Social; 2013.

\section{Notas}

* Artículo de investigación.

\section{Licencia Creative Commons CC BY 4.0}

Cómo citar este artículo: Escolano Rivas J, Barrientos Sánchez S, Rodríguez Ciódaro A. Frecuencia, hallazgos y variaciones óseas en radiografías panorámicas de personas con edentulismo total. Univ Odontol. 2018 enejun; 37(78). https://doi.org/10.11144/Javeriana.uo37-78.fhvo 